Supplement of Geosci. Model Dev., 13, 2511-2532, 2020

https://doi.org/10.5194/gmd-13-2511-2020-supplement

(C) Author(s) 2020. This work is distributed under

the Creative Commons Attribution 4.0 License.

(c) (1)

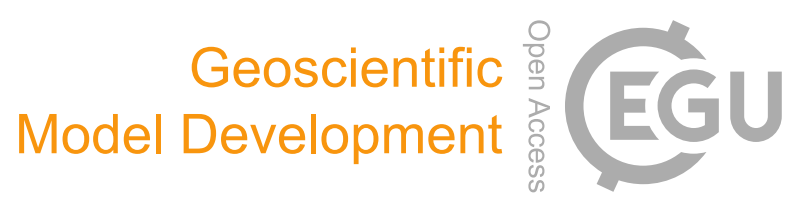

Supplement of

\title{
Investigating the sensitivity to resolving aerosol interactions in downscal- ing regional model experiments with WRFv3.8.1 over Europe
}

Vasileios Pavlidis et al.

Correspondence to: Vasileios Pavlidis (vapavlid@physics.auth.gr)

The copyright of individual parts of the supplement might differ from the CC BY 4.0 License. 


\section{Supplement}

Table S1. Domain averaged precipitation difference $(\mathrm{mm} /$ day) and relative difference (\%) from CON. For all experiments and seasons. Where stated, for simulation $A R C l$ the above quantities are also calculated against $A C l(A R C l-A C l)$ in order to assess the implementation of aerosol-radiation interactions in the Thompson aerosol-cloud interacting cloud microphysics.

\begin{tabular}{|c|c|c|c|c|c|c|c|c|}
\hline & \multicolumn{2}{|c|}{ DJF } & \multicolumn{2}{|c|}{ MAM } & \multicolumn{2}{|c|}{ JJA } & \multicolumn{2}{|c|}{ SON } \\
\hline & $\mathrm{mm} /$ day & relative $\%$ & $\mathrm{~mm} /$ day & relative $\%$ & $\mathrm{~mm} / \mathrm{day}$ & relative $\%$ & $\mathrm{~mm} / \mathrm{day}$ & relative $\%$ \\
\hline ARI_T & 0,00 & 0 & $-0,08$ & -4 & $-0,08$ & -5 & 0,04 & 2 \\
\hline ARI_Mv1 & 0,00 & 0 & $-0,05$ & -2 & $-0,02$ & -2 & 0,00 & 0 \\
\hline ARI_Mv1urban & $-0,05$ & -2 & $-0,24$ & -13 & $-0,21$ & -14 & $-0,06$ & -2 \\
\hline ARI_Mv1full & $-0,01$ & 0 & $-0,01$ & 0 & 0,00 & 0 & 0,06 & 3 \\
\hline ARI_MC & 0,01 & 0 & $-0,09$ & -5 & $-0,03$ & -2 & 0,02 & 1 \\
\hline $\mathrm{ARCl}-\mathrm{ACl}$ & $-0,04$ & -1 & 0,02 & 1 & $-0,03$ & -2 & $-0,13$ & -5 \\
\hline $\mathrm{ACl}$ & $-0,04$ & -2 & $-0,05$ & -3 & 0,00 & 0 & 0,07 & 3 \\
\hline $\mathrm{ARCl}$ & $-0,08$ & -3 & $-0,03$ & -2 & $-0,04$ & -2 & $-0,06$ & -2 \\
\hline
\end{tabular}

Table S2. Relative difference (\%) from control CON for shortwave radiation (Rsds), direct normalized irradiance (DNI) and diffuse radiation at the surface (DIF). Where stated, for simulation $A R C l$ the above quantities are also calculated against $A C l$ (ARCI-ACI) in order to assess the implementation of aerosol-radiation interactions in the Thompson aerosol-cloud interacting cloud microphysics. For all simulations and seasons.

\begin{tabular}{|c|c|c|c|c|c|c|c|c|c|c|c|c|}
\hline & \multicolumn{3}{|c|}{ DJF } & \multicolumn{3}{|c|}{ MAM } & \multicolumn{3}{|c|}{ JJA } & \multicolumn{3}{|c|}{ SON } \\
\hline & Rsds & DNI & DIF & Rsds & DNI & DIF & Rsds & DNI & DIF & Rsds & DNI & DIF \\
\hline ARI_T & -5 & -30 & 7 & -4 & -22 & 12 & -7 & -27 & 36 & -8 & -31 & 16 \\
\hline ARI_Mv1 & -5 & -33 & 8 & -5 & -29 & 17 & -6 & -30 & 38 & -7 & -34 & 18 \\
\hline ARI_Mv1urban & -11 & -33 & -3 & -12 & -29 & 0 & -13 & -29 & 12 & -16 & -37 & 1 \\
\hline ARI_Mv1full & -4 & -31 & 9 & -5 & -28 & 17 & -7 & -29 & 37 & -6 & -33 & 18 \\
\hline ARI_MC & -3 & -26 & 7 & -5 & -27 & 15 & -6 & -29 & 35 & -7 & -29 & 15 \\
\hline $\mathrm{ARCl}-\mathrm{ACl}$ & -2 & -20 & 6 & -4 & -23 & 12 & -6 & -25 & 26 & -3 & -18 & 14 \\
\hline $\mathrm{ACl}$ & 7 & 8 & 7 & 7 & 9 & 5 & 9 & 11 & 6 & 8 & 6 & 7 \\
\hline $\mathrm{ARCl}$ & 5 & -14 & 13 & 2 & -16 & 17 & 2 & -17 & 31 & 5 & -14 & 21 \\
\hline
\end{tabular}




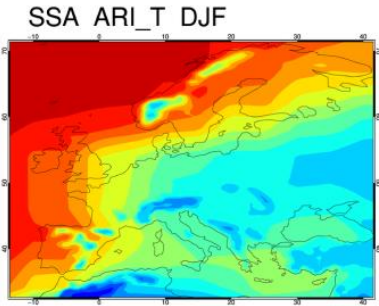

SSA ARI_Mv1 DJF

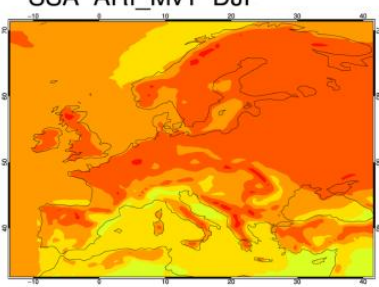

SSA ARI_Mv1full DJF
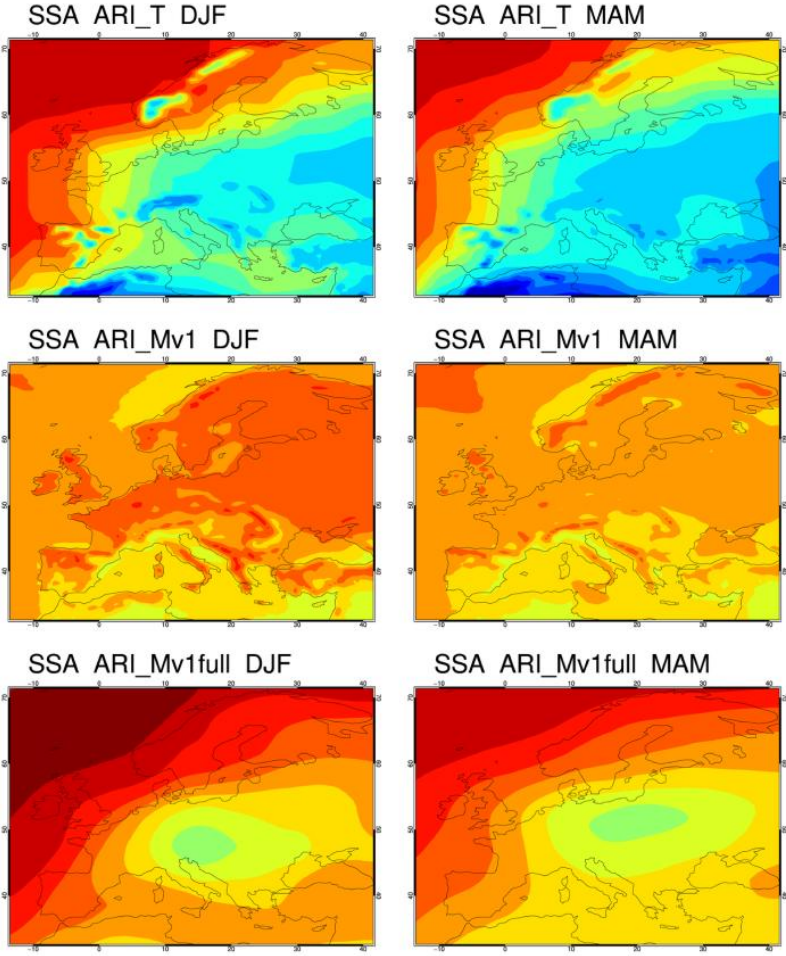

SSA ARI Mv1 MAM

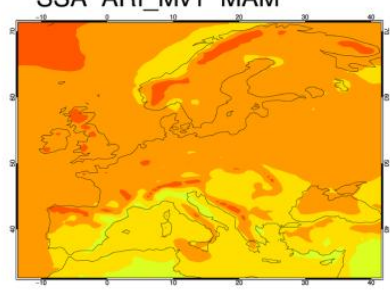

SSA ARI Mv1full MAM
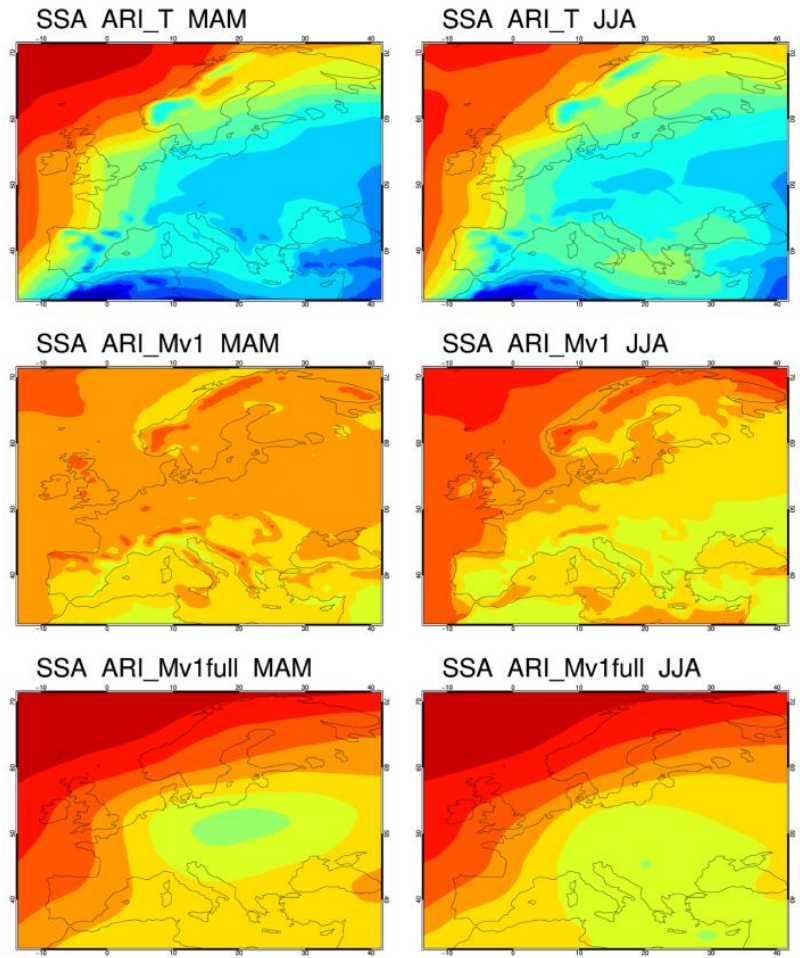

SSA ARI_T SON

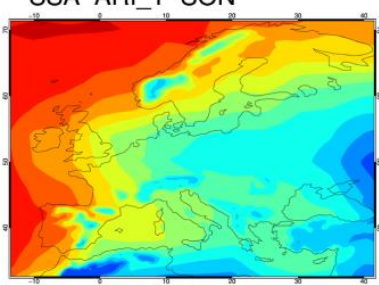

SSA ARI Mv1 JJA

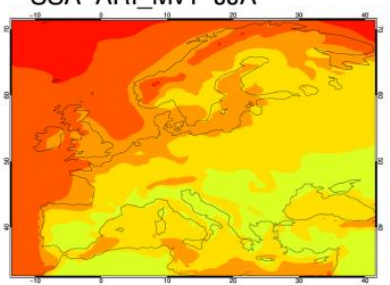

SSA ARI Mv1full JJA

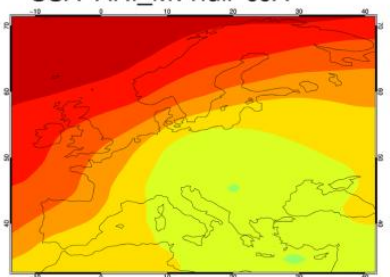

SSA ARI Mv1 SON

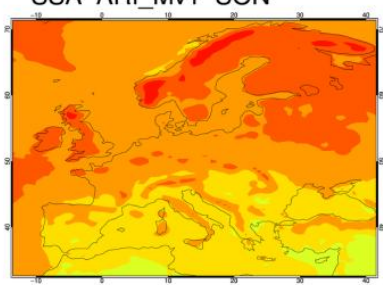

SSA ARI_Mv1full SON

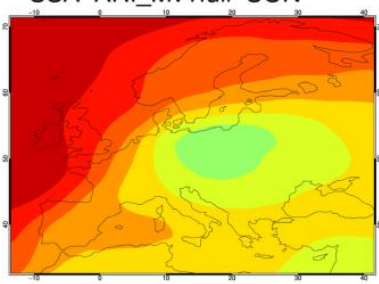

\section{$\begin{array}{lllllllll}0.84 & 0.86 & 0.88 & 0.90 & 0.92 & 0.94 & 0.96 & 0.98 & 1.00\end{array}$}
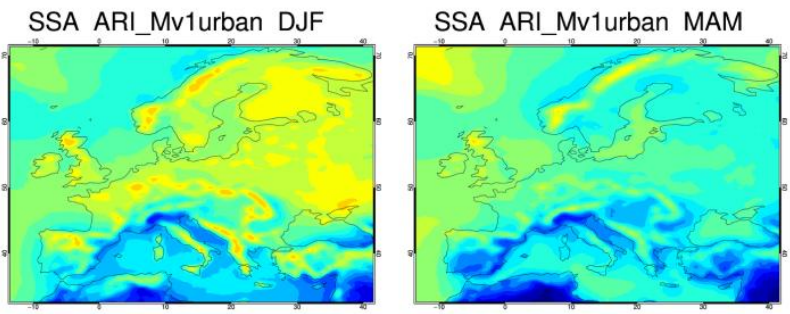

SSA ARI_Mv1urban JJA

SSA ARI_Mv1urban SON
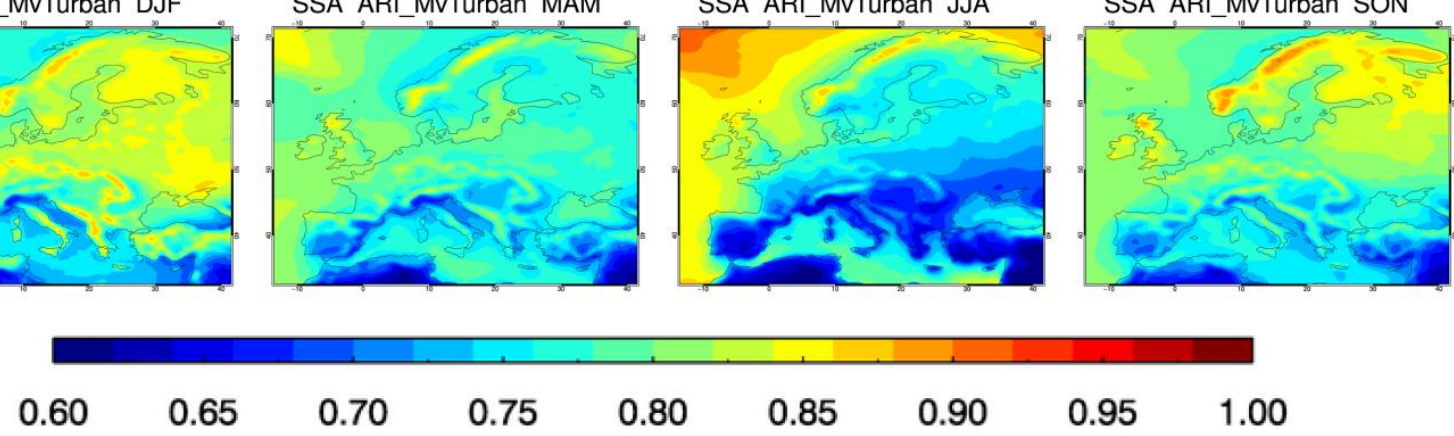

0.70

0.75

0.80

0.85

0.90

$0.95 \quad 1.00$

Figure S1: Single scattering albedo averaged values for the spectral range 0.4-1 $\mu$ m. For simulations ARI_T (aer_opt=1 option), ARI_Mv1 (aer_opt=2 and "rural" aerosol type) and ARI_Mv1full (MAC-v1 SSA climatology). At the bottom row with a different color scale for the ARI_Mv1urban simulation (aer_opt=2 and "urban" aerosol type). 

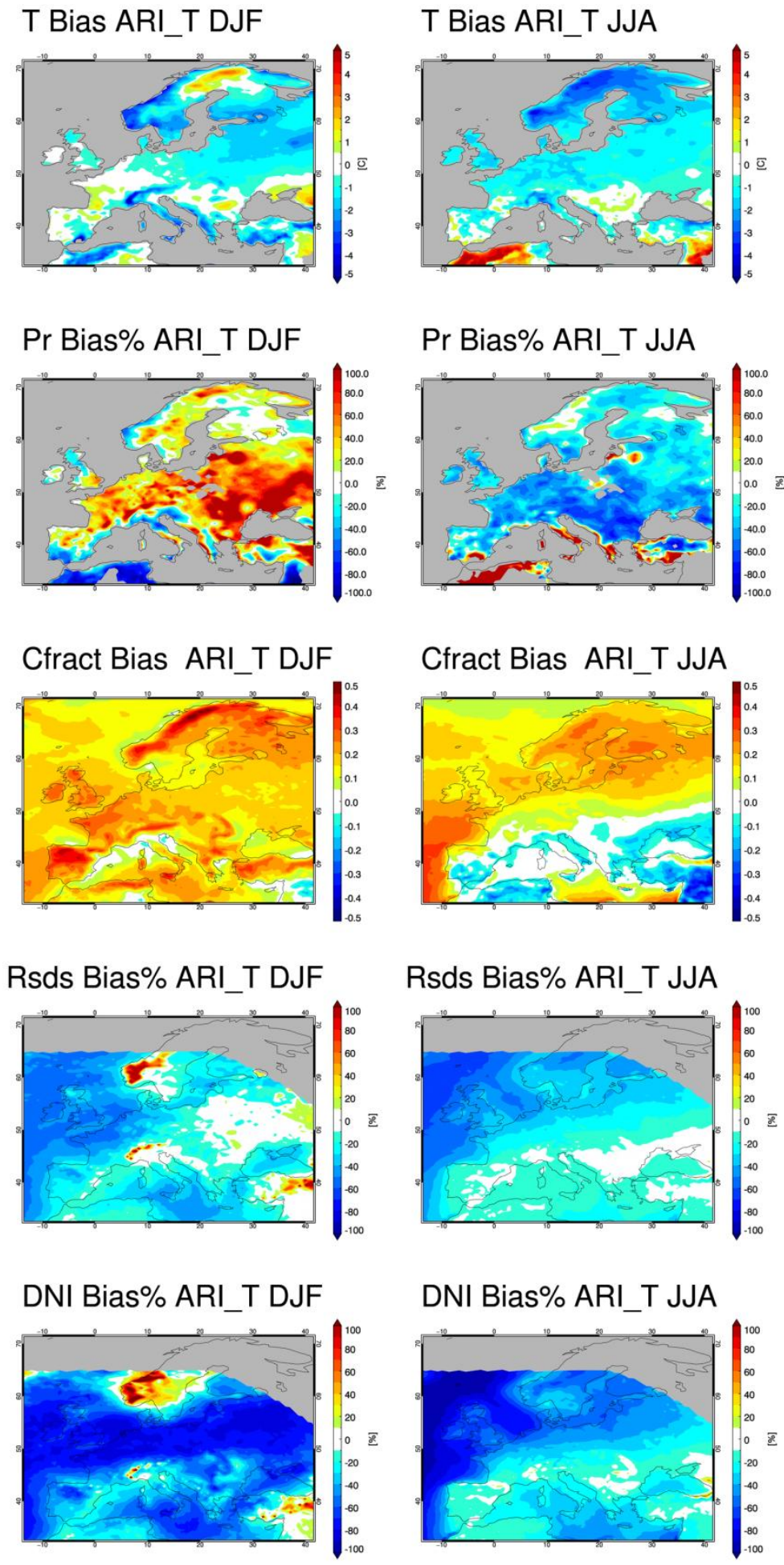

Figure S2: Bias plots for control simulation ARI_T for winter (DJF-left) and summer (JJA-right). Biases depicted from top to bottom for temperature (T), precipitation (Pr), total cloud fraction (Cfract), down welling shortwave radiation to the surface (Rsds) and direct normalized irradiance at the surface (DNI). 


\section{Rsds difference\%}

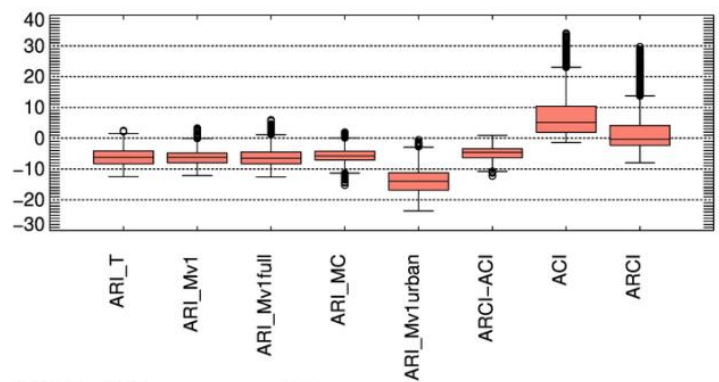

DNI difference \%

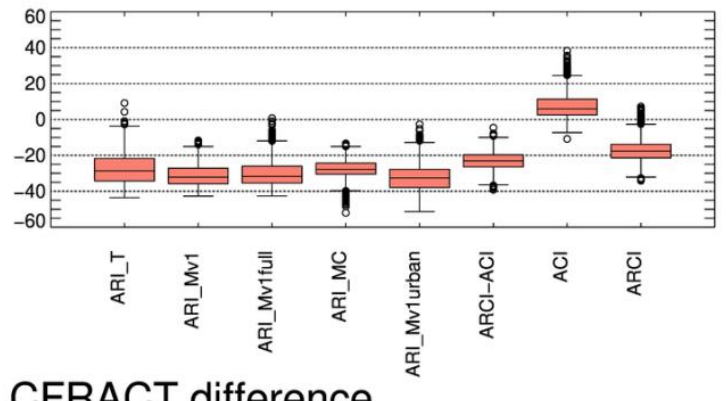

CFRACT difference

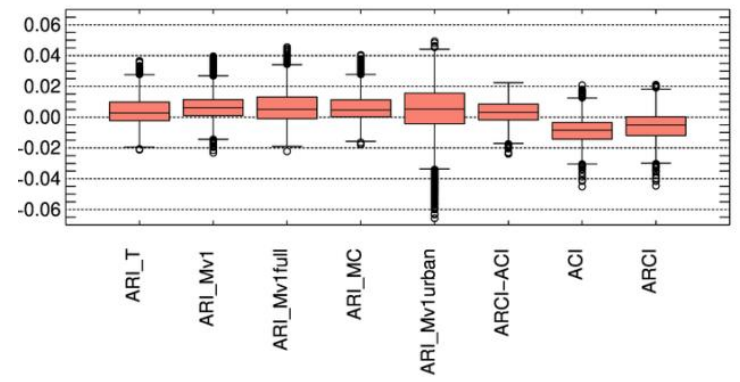

\section{DIF difference\%}

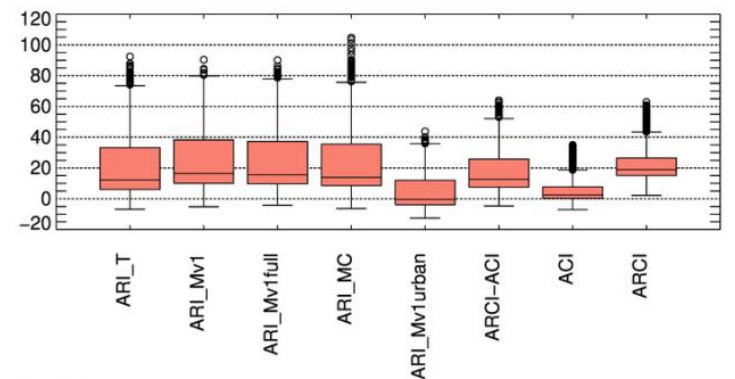

T difference

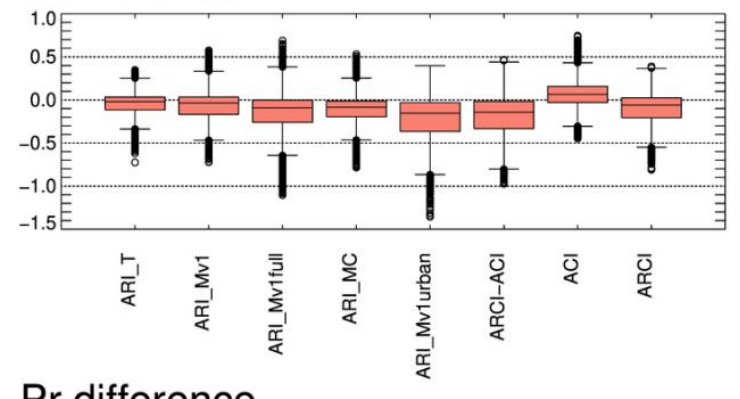

Pr difference

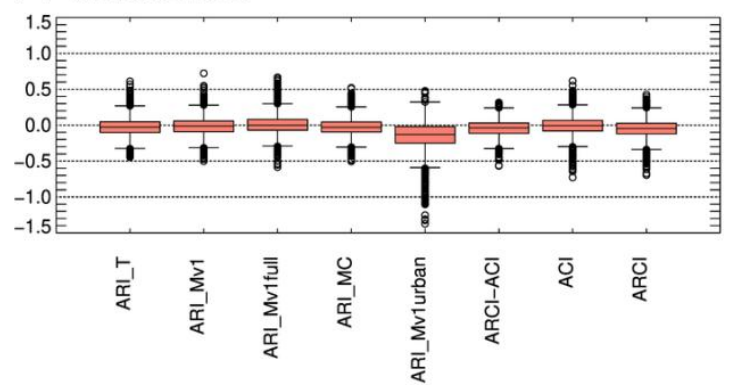

Figure S3: Plots of differences of the aerosol including simulations from control CON regarding the grid points of the domain on a yearly mean basis. For shortwave radiation at the surface (Rsds), diffuse radiation at the surface (DIF) and direct normalized irradiance (DNI), temperature $(T)$, total cloud fraction CFRACT) and precipitation(Pr). Where stated, the difference of simulation $A R C l$ to $A C l$ is given (ARCI-ACI) to assess the implementation of aerosol-radiation interactions in the Thompson aerosol-cloud interacting cloud microphysics. 
Wind 850 CON DJF

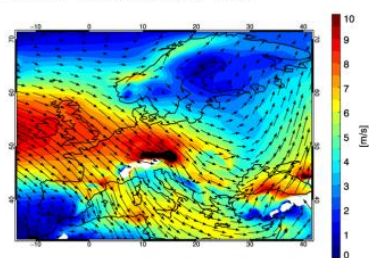

Wind 850 ARI_T-CON DJF

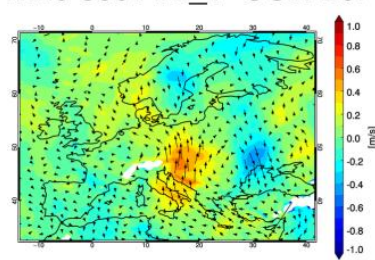

Wind 850 CON MAM

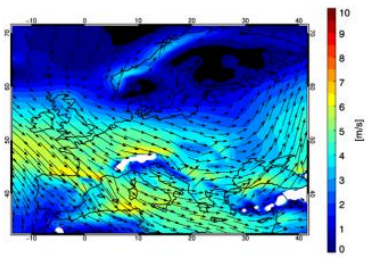

Wind 850 CON JJA

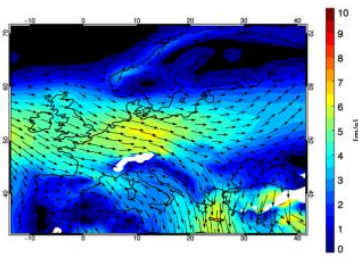

Wind 850 CON SON

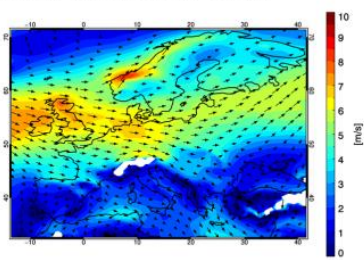

Wind 850 ARI_T-CON MAM Wind 850 ARI_T-CON JJA Wind 850 ARI_T-CON SON
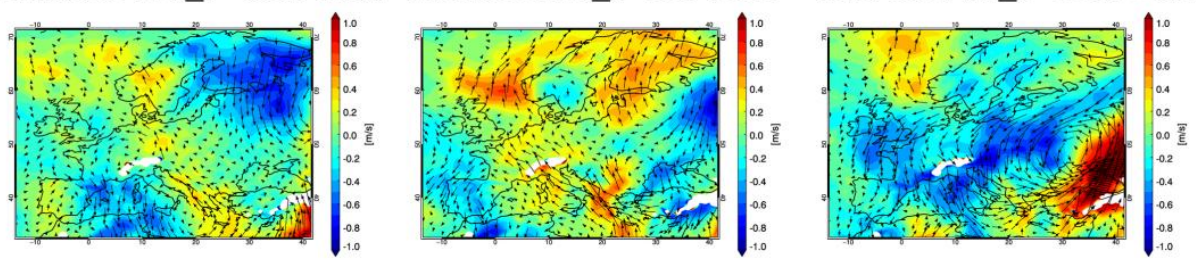

Wind 500 ARI_T-CON DJF

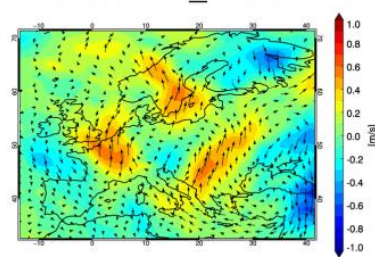

Wind 500 ARI T-CON MAM Wind 500 ARI T-CON JJA

Wind 500 ARI_T-CON SON
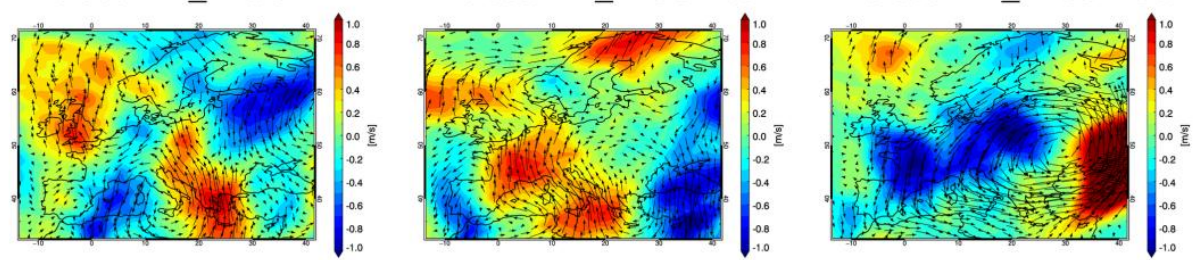

Figure S4: First row depicts the wind field (arrows) and wind speed (colored contours) of the control experiment for each season. Second row depicts the wind field anomalies (arrows) and meridional wind speed difference (colored contours) of simulation ARI_T from control CON at 850hPa. Likewise for the 500hPa level at the bottom row. Stippling indicates areas where the differences are statistically significant at the $95 \%$ level, according to the Mann-Whitney non parametric test. 
AOD Tegen SON

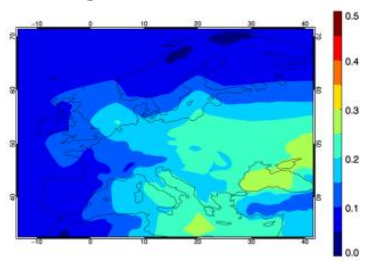

T ARI_T-CON 48.25lat

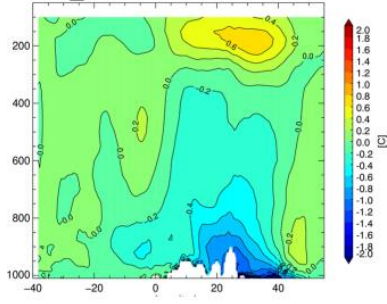

Wind 850 ARI_T-CON

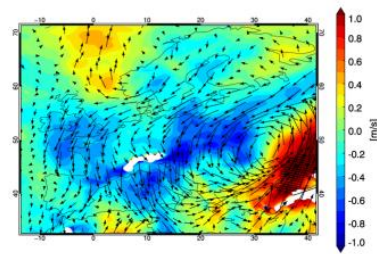

RE ARI_T-CON

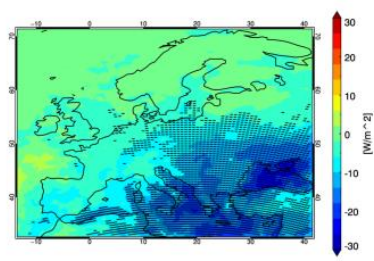

CFRACT ARI_T-CON

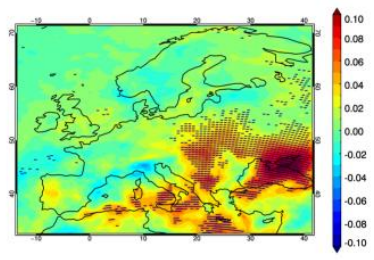

Wind 500 ARI_T-CON

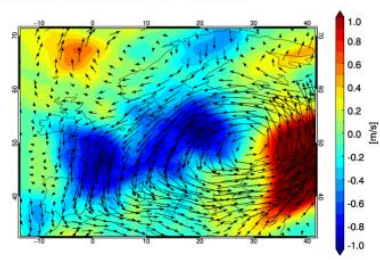

T ARI T-CON

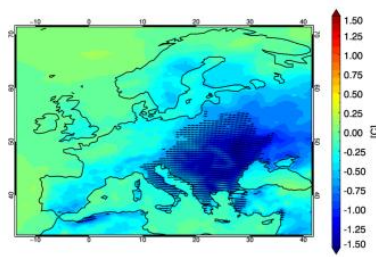

$\triangle$ SCRE ARI_T-CON

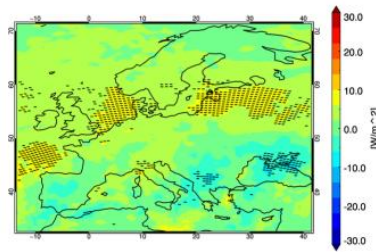

Wind w 850 ARI_T-CON

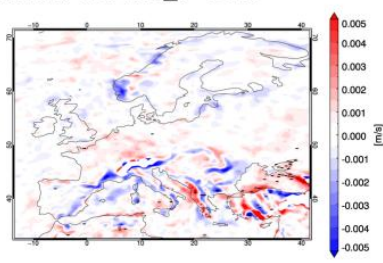

T 850 ARI T-CON

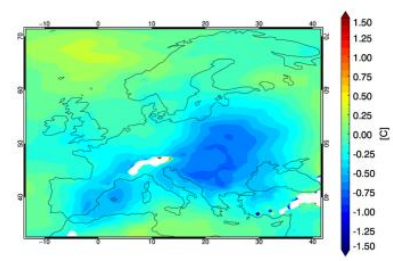

Pr ARI_T-CON \% SON

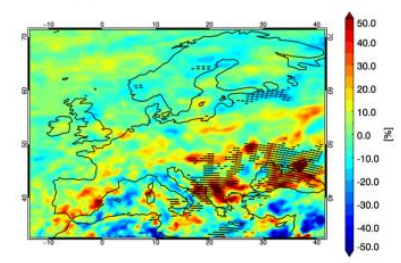

Wind w 500 ARI_T-CON

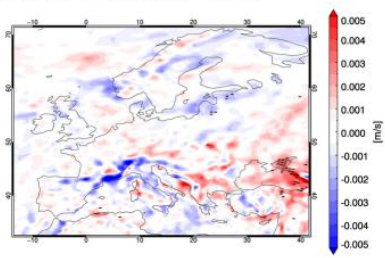

Figure S.5: Composite analysis for simulation ARI_T in SON. All differences are calculated from CON. Top row, from left to right: AOD field, Radiative forcing RE, difference in near-surface temperature (T), difference in temperature at the 850hPa level (T850). Second row: cross-section at 48.25 latitude depicting differences in temperature vertical profile, difference in cloud fraction (CFRACT), differences in cloud forcing (SCRE), relative (\%) difference in precipitation (Pr), Third row: differences in the wind field (arrows) and meridional wind speed (colored contours) at $850 \mathrm{hPa}$, differences in the wind field (arrows) and meridional wind speed (colored contours) at 500hPa, difference in vertical wind speed w at $850 \mathrm{hPa}$ and at 500hPa. Stippling indicates areas of statistical significance. 
AOD Macv1 JJA

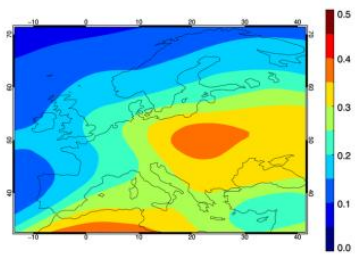

T ARI_Mv1urban-CON

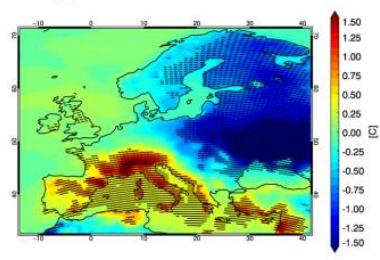

Wind 850 ARI_Mv1urban-CON

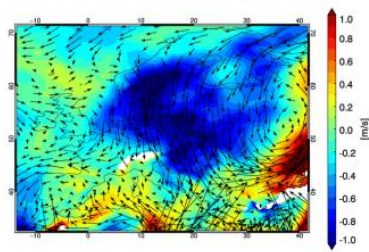

RE ARI Mv1urban-CON

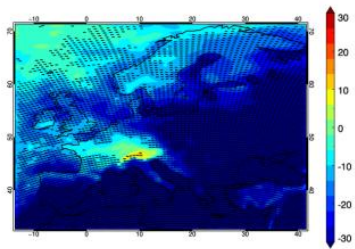

T 850 ARI Mv1urban-CON JJA

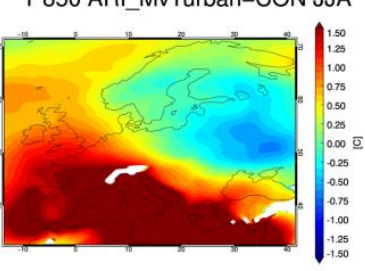

Wind 500 ARI_Mv1urban-CON

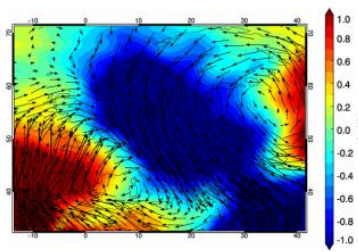

CFRACT ARI Mv1urban-CON
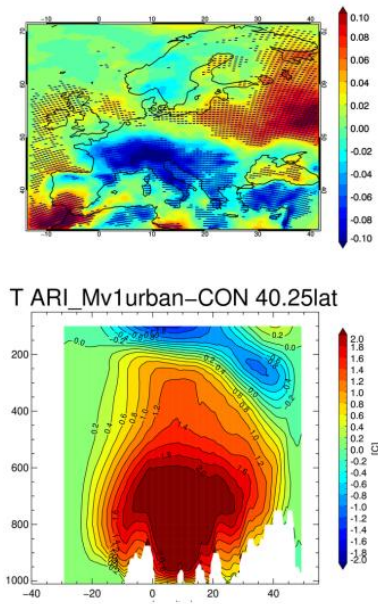

Wind w 850 ARI Mv1urban-CON

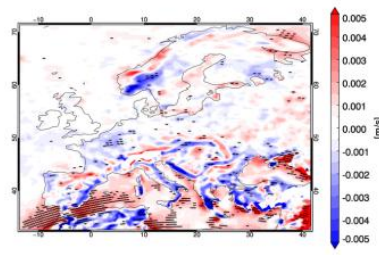

$\triangle$ SCRE ARI Mv1urban-CON

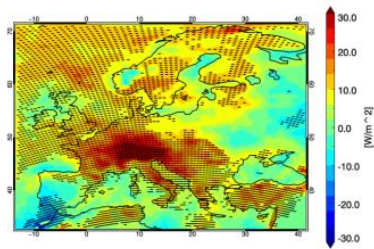

T ARI_Mv1urban-CON 52.25lat

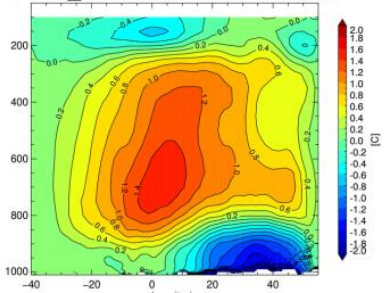

Wind w 500 ARI_Mv1urban-CON

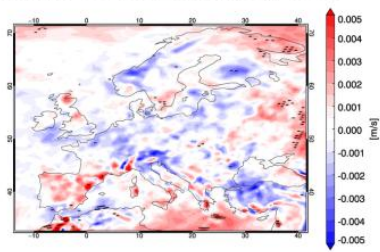

Figure S6: Composite analysis for simulation ARI_Mv1urban in JJA. All differences are calculated from CON. Top row, from left to right: AOD field, Radiative forcing (RE), difference in cloud fraction (CFRACT), difference in cloud forcing (SCRE). Second row: Difference in near-surface temperature $(T)$, temperature differences at $850 \mathrm{hPa}$ (T850), cross-section at 40.25 latitude depicting differences in temperature vertical profile, cross-section at 50.25 latitude depicting differences in temperature vertical profile. Bottom row: difference in the wind field (arrows) and meridional wind speed (colored contours) at $850 \mathrm{hPa}$ and 500hPa, difference in vertical wind speed w at $850 \mathrm{hPa}$ and at 500hPa. Stippling indicates areas of statistical significance.
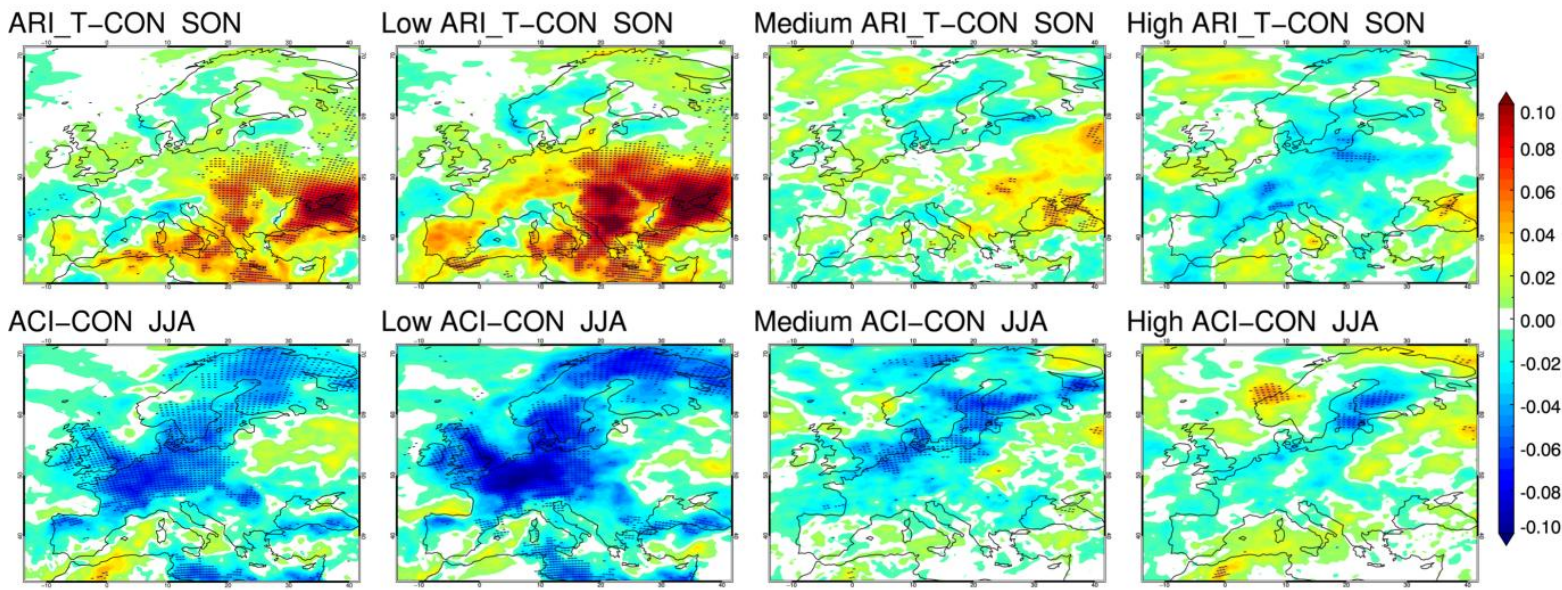
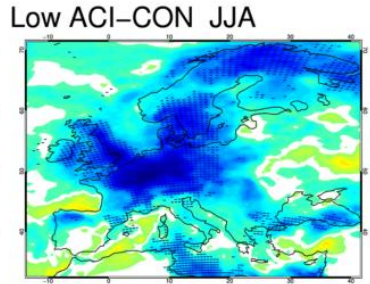
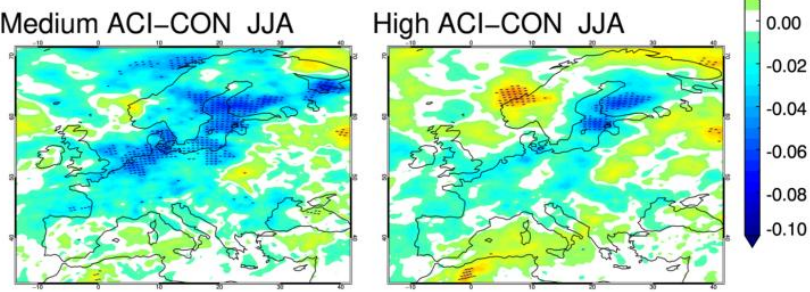

Figure S.7: Difference from control simulation CON regarding total cloud fraction (first column) as well as cloud fraction for Low, Medium and High clouds (second to fourth column respectively) for simulation ARI_T during Autumn (top row) and simulation ACl during summer (bottom row). Stippling indicates areas where the differences are statistically significant at the 95\% level, according to the Mann-Whitney non parametric test. 


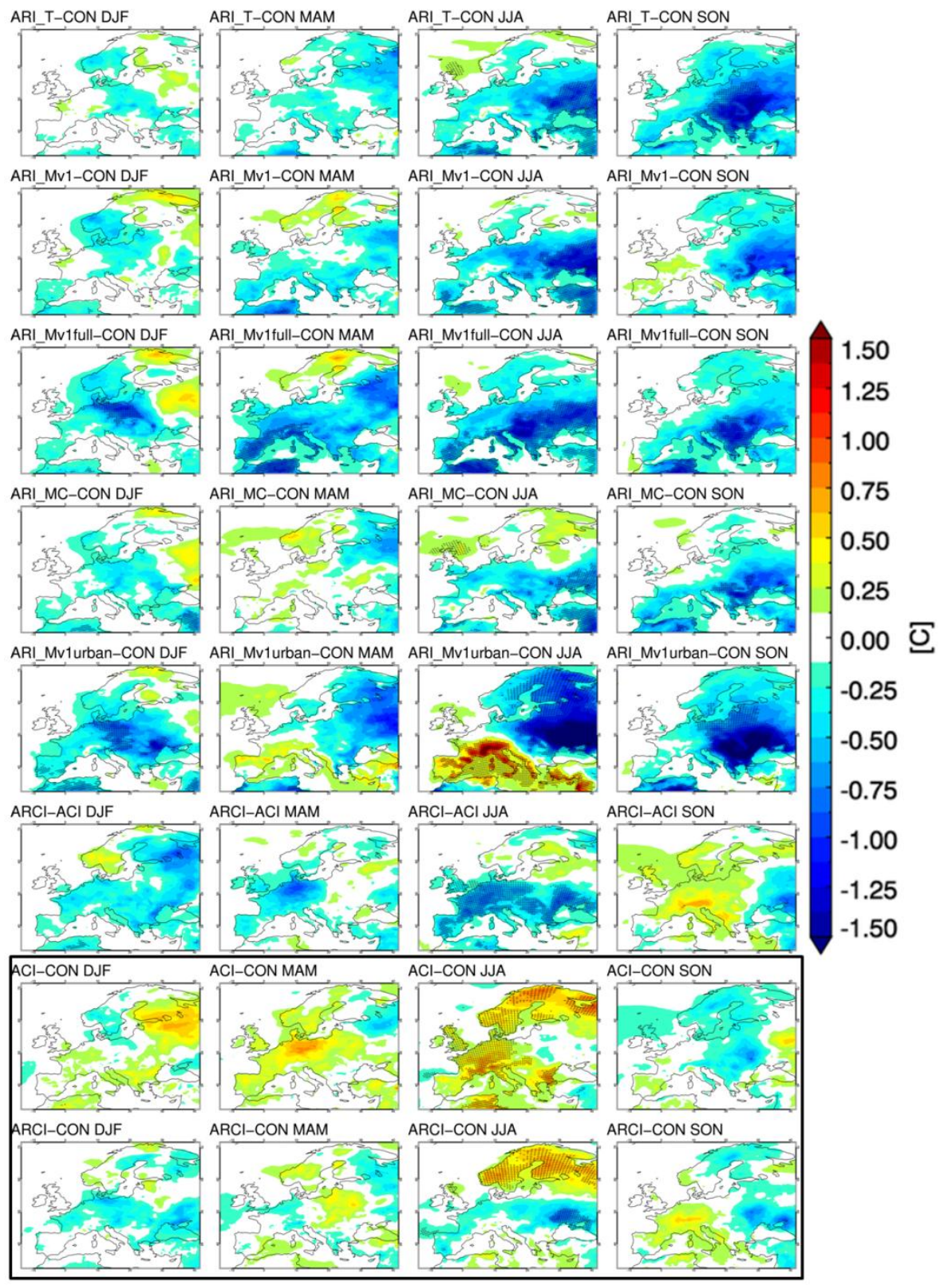

Figure S8: Near-surface temperature (T) changes from control simulation CON for all experiments and seasons. First five rows present the impact of aerosol-radiation interactions only. Furthermore the temperature difference of $A R C l$ calculated against $A C l(A R C l-A C l)$ is given to assess the implementation of aerosol-radiation interactions in the Thompson aerosol-interacting cloud microphysics (TE2014) that enables aerosol-cloud interactions (row six). Last two rows (black box) present the impact of TE2014 against control CON (row seven, ACI-CON) and TE2014 with aerosol-radiation interactions enabled against control CON (row eight, ARCI-CON). Stippling indicates areas where the differences are statistically significant at the $95 \%$ level, according to the Mann-Whitney non parametric test. 


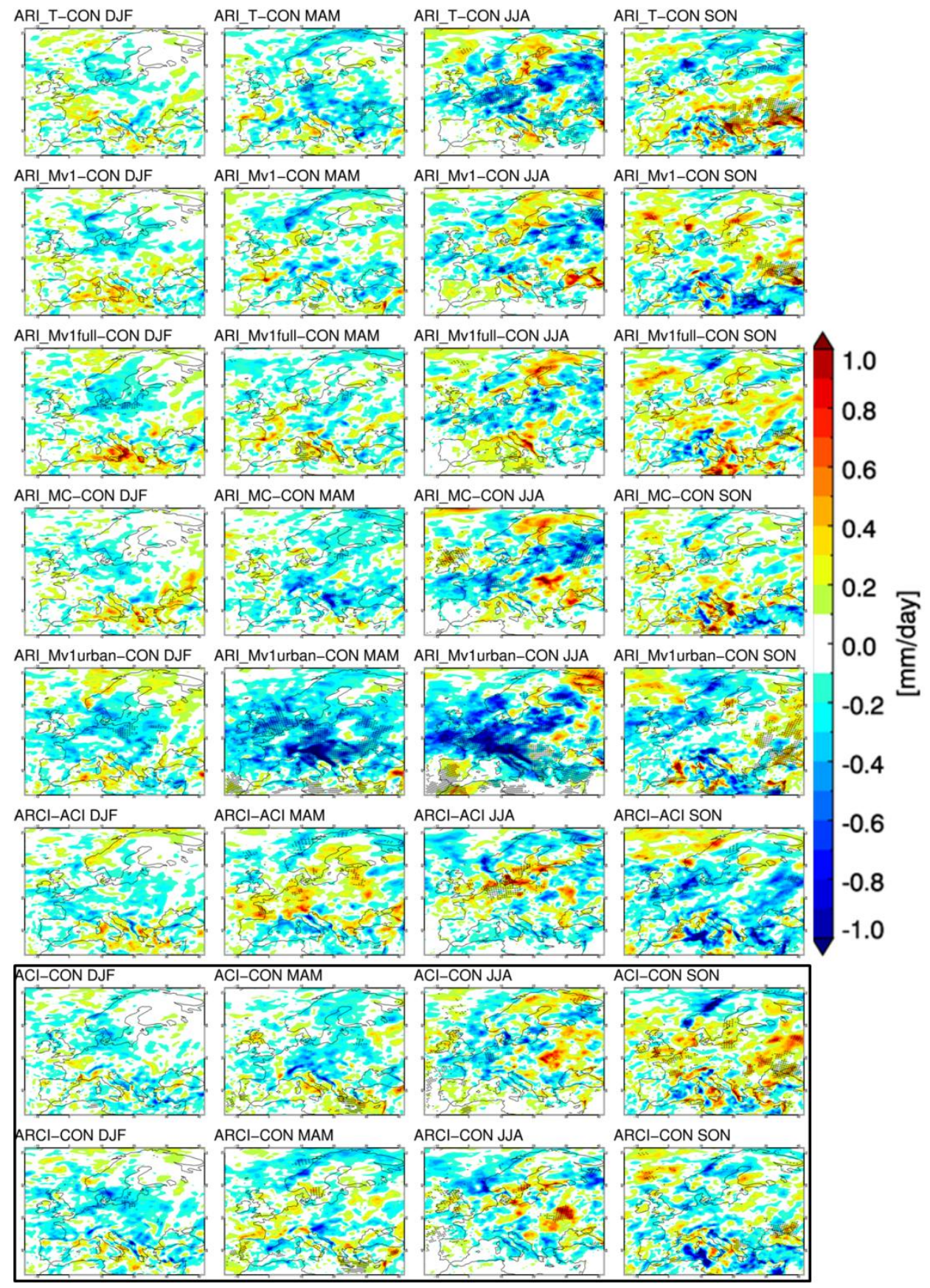

Figure S9: Precipitation difference ( $\mathrm{mm} /$ day) from control simulation CON for all experiments and seasons. First five rows present the impact of aerosol-radiation interactions only. Furthermore the temperature difference of $A R C l$ calculated against $A C l(A R C l-A C l)$ is given to assess the implementation of aerosol-radiation interactions in the Thompson aerosol-interacting cloud microphysics (TE2014) that enables aerosol-cloud interactions (row six). Last two rows (black box) present the impact of TE2014 against control CON (row seven, ACI-CON) and TE2014 with aerosol-radiation interactions enabled against control CON (row eight, ARCI-CON). Stippling indicates areas where the differences are statistically significant at the $95 \%$ level, according to the Mann-Whitney non parametric test. 